\title{
Degree of Polarization Degradation Due to Cross-Phase Modulation and Its Impact on Polarization-Mode Dispersion Compensators
}

\author{
Alberto Bononi, Armando Vannucci, Member, IEEE, A. Orlandini, Member, IEEE, E. Corbel, S. Lanne, and \\ S. Bigo, Member, IEEE
}

\begin{abstract}
A novel analytical model is proposed to predict the cross-phase modulation (XPM)-induced depolarization in a two-channel transmission system, in which the Stokes' vector of each channel rotates around a space-invariant pivot by a time-varying angle which depends on the total instantaneous optical power in the fiber, on the angle between pump and probe input Stokes' vectors, and on the walk-off between channels. The model leads to a simple formula of the probe degree of polarization (DOP) which is validated both by simulation and experiment. The model helps identify the key physical factors that determine the XPM-induced performance degradation of DOP-based first-order polarization-mode dispersion compensators, and experiments that quantify such degradation are presented.
\end{abstract}

Index Terms-Nonlinearity, optical fibers, optical systems, polarization, Stokes' parameters, wavelength-division multiplexing (WDM).

\section{INTRODUCTION}

$\mathbf{I}$ $\mathrm{N}$ A wavelength-division-multiplexed (WDM) system, the nonlinear effect of cross-phase modulation (XPM) induces a rapid change of the state of polarization (SOP) on each channel [1], even when polarization-mode dispersion (PMD) is not present. Such a nonlinear polarization effect, which depends on the total instantaneous optical power in the fiber, produces a time-dependent polarization scattering on the time scale of a single bit. The ensuing depolarization of the WDM channels may pose a serious limitation on those links where optical polarization-mode dispersion compensators (OPMDC) are needed to reduce the PMD-induced system impairments. Such compensators, in fact, are designed to compensate a single WDM channel independently of the others, in the linear regime of propagation. Most of them [2]-[4] typically use a polarization controller, a polarization-maintaining fiber (PMF) with a fixed delay, and a feedback control signal, e.g., the degree of polarization (DOP) [2] or a linear combination of spectral lines of the output signal [3], [4]. The optimum coupling condition between the line and the compensator is found by monitoring the feedback signal, which is assumed to change slowly on the PMD millisecond time scale. However, OPMDCs cannot mitigate the rapid distorting effects of XPM which appear on the feedback control on the bit time scale of

Manuscript received January 29, 2003; revised May 2, 2003.

A. Bononi, A. Vannucci, and A. Orlandini are with the Dipartimento di Ingegneria dell'Informazione, Università degli Studi di Parma, I-43100 Parma, Italy.

E. Corbel, S. Lanne, and S. Bigo are with the Alcatel Research and Innovation, Route de Nozay, F-91460 Marcoussis, France.

Digital Object Identifier 10.1109/JLT.2003.815513 fractions of nanoseconds. As shown in some recent experiments [5], [7]-[9], when XPM-induced degradation is present, the efficiency of OPMDCs is drastically reduced.

Moreover, with the aim of predicting such efficiency reduction, the study of depolarization due to the interaction of Kerr nonlinearity and PMD has been tackled in several experimental and theoretical works [10]-[12]. In [10], the focus is on the analysis by simulation of the depolarization induced by self-phase modulation (SPM) and its dependence on the transmitted pulse chirp. Möller et al. [11] have experimentally shown that if the SOPs of the WDM channels are launched in such a way that the overall input DOP is minimized, the effects of XPM are also minimized and the output DOP is maximized.

The main theoretical result so far available was obtained by Wang and Menyuk [1] in the assumption of a significant walk-off among the WDM channels, so that each channel senses only the continuous-wave $(\mathrm{CW})$ component of the remaining WDM channels. Thus, the coupled nonlinear Schroedinger equation (CNLSE) in the absence of both PMD and group velocity dispersion (GVD) can be solved exactly, and it is concluded in [1] that the SOP change of each channel is space dependent, but the DOP of each channel is not degraded. With the same assumptions, Collings and Boivin [12] have tackled the two-channel case, showing that the analytical solution can be interpreted as a rotation of the pump and probe Stokes' vectors around a fixed pivot during propagation.

However, when a finite walk-off is taken into account, the modulation of the channels rules out an exact closed-form solution of the CNLSE, so that no fixed pivot around which SOP vectors rotate can be identified anymore.

In this paper, we present a simplified approximate model for a two-channel WDM system where both channel modulation and walk-off are accounted for, and we apply it to the evaluation of the DOP in fibers with negligible PMD. According to the proposed model, the Stokes' vector of each channel rotates around a space-invariant pivot by a time-varying angle which depends on the total instantaneous optical power in the fiber, on the angle between pump and probe input Stokes' vectors, and on the walk-off between channels. The fixed pivot is the vector sum of the probe and pump Stokes' vectors.

The model assumes a continuous-wave probe and leads to an explicit formula for the DOP, which is based on the walk-off-induced linear filtering of the pump channel, similarly to related well-known results in the analysis of both XPM in scalar nonlinear propagation [13], [14] and of pump-induced relative intensity noise in Raman amplifiers [15], [16]. 
The DOP formula turns out to be quite simple when the pump is ON-OFF keying (OOK) modulated by a square wave emulating an infinite alternation of marks and spaces, while it is slightly more complex with random pump bit sequences (RBS). A good agreement of the DOP formula with $10-\mathrm{Gb} / \mathrm{s}$ experimental results and simulations based on the beam propagation method (BPM) [17] is found. Simulation results are also presented that show the DOP degradation in the presence of PMD.

We carried out an experiment of a two-channel $10-\mathrm{Gb} / \mathrm{s}$ WDM system whose statistical performance in the presence of XPM was studied both with and without first-order DOP-based PMD compensation [8]. The results show that PMD distorting effects are emphasized by XPM, and therefore the OPMDC efficiency is reduced.

The paper is organized as follows. In Section II, the novel analytical model for XPM-induced DOP degradation in a WDM system is derived for a pump and probe scheme. In Section III, the DOP of the probe is computed from a linear filtering of the pump channel, and an explicit DOP formula in the case of a 1010 modulated pump, and for an RBS modulated pump are derived and checked against BPM simulations. In Section IV, experimental DOP measurements for varying launch power conditions are shown in order to validate the analytical DOP formula. Moreover, the performance of a WDM system with PMD and XPM is evaluated in terms of the statistics of the $Q$-factor penalty, both with and without first-order PMD compensation. In Section V, we summarize our conclusions. The appendix contains analytical results used in Section III.

\section{The CARousel Model}

In a long WDM system with negligible PMD, the evolution of each propagating channel in the nonlinear regime can be effectively described by the Manakov equation [1], which takes into account the interaction between the nonlinearity and the rapidly varying fiber birefringence through a scaling of the nonlinear coefficient $\gamma$ by a factor $8 / 9$. Such equation is obtained from the CNLSE by averaging the Kerr effect over the Poincaré sphere under the assumption of complete mixing, which is verified when the fiber correlation length is much shorter than the nonlinear length [18].

The Manakov equation for two completely polarized OOKmodulated probe and pump signals, propagating at wavelengths $\lambda_{s}$ and $\lambda_{p}$, yields the following system of nonlinear differential equations $^{1}$ in the probe retarded-time frame $t$ along the fiber length $z[1]$

$$
\left\{\begin{array}{c}
\frac{\partial \underline{S}(z, t)}{\partial z}-j \frac{\beta_{2 s}}{2} \frac{\partial \underline{S}^{2}(z, t)}{\partial t^{2}} \\
+j \frac{8}{9} \gamma e^{-\alpha z}[\underbrace{\left(\underline{S}^{\dagger} \underline{S}\right) \underline{S}}_{\mathrm{SPM}}+\underbrace{\left(\underline{P}^{\dagger} \underline{P}\right) \underline{S}+\left(\underline{P}^{\dagger} \underline{S}\right) \underline{P}}_{\mathrm{XPM}}]=0 \\
\frac{\partial \underline{P}(z, t)}{\partial z}+d_{s p} \frac{\partial \underline{P}(z, t)}{\partial t}-j \frac{\beta_{2 p}}{2} \frac{\partial \underline{P}^{2}(z, t)}{\partial t^{2}} \\
+j \frac{8}{9} \gamma e^{-\alpha z}\left[\left(\underline{P}^{\dagger} \underline{P}\right) \underline{P}+\left(\underline{S}^{\dagger} \underline{S}\right) \underline{P}+\left(\underline{S}^{\dagger} \underline{P}\right) \underline{S}\right]=0
\end{array}\right.
$$

where $\underline{S}(z, t)$ and $\underline{P}(z, t)$ are the complex envelopes of probe and pump, respectively, referred to the probe frequency; $d_{s p} \simeq$ $D_{c}\left(\lambda_{s}-\lambda_{p}\right)$ is the walk-off parameter [17]; $\beta_{2 s}$ and $\beta_{2 p}$ are the

\footnotetext{
${ }^{1}$ We use here the engineers' notation for Fourier transforms, as opposed to [1], where the physicists' notation is used.
}

GVD coefficients at $\lambda_{s}$ and $\lambda_{p}$, respectively; and the symbol ${ }^{\dagger}$ indicates the transpose conjugate. Note that the envelopes are unattenuated, as attenuation has been removed by a change of variable and appears as an exponential that multiplies the nonlinear coefficient $\gamma$. In the first equation in (1), we recognize that $\left(\underline{P}^{\dagger} \underline{S}\right) \underline{P}=\left(\underline{P} \underline{P}^{\dagger}\right) \underline{S}=\left((1 / 2) \sum_{i=0}^{3} p_{i} \sigma_{i}\right) \underline{S}$, where the $\sigma_{i}$ 's are the Pauli matrices and we used the standard decomposition of the projector matrix $\underline{P} \underline{P}^{\dagger}$ [19], in which the pump intensity $p_{0}(z, t)=\underline{P}^{\dagger} \underline{P}$ and the instantaneous pump Stokes' vector $\vec{p}(z, t)=\left(p_{1}, p_{2}, p_{3}\right)$ appear. By using similar manipulations involving the Stokes' vector of the probe, (1) becomes

$$
\left\{\begin{array}{l}
\frac{\partial \underline{S}(z, t)}{\partial z}-j \frac{\beta_{2 s}}{2} \frac{\partial \underline{S}^{2}(z, t)}{\partial t^{2}} \\
\quad+j \frac{8}{9} \gamma e^{-\alpha z}\left[s_{0} \sigma_{0}+\frac{3}{2} p_{0} \sigma_{0}+\frac{1}{2} \vec{p} \cdot \vec{\sigma}\right] \underline{S}(z, t)=0 \\
\frac{\partial P(z, t)}{\partial z}+d_{s p} \frac{\partial P(z, t)}{\partial t}-j \frac{\beta_{2 p}}{2} \frac{\partial P^{2}(z, t)}{\partial t^{2}} \\
\quad+j \frac{8}{9} \gamma e^{-\alpha z}\left[p_{0} \sigma_{0}+\frac{3}{2} s_{0} \sigma_{0}+\frac{1}{2} \vec{s} \cdot \vec{\sigma}\right] \underline{P}(z, t)=0
\end{array}\right.
$$

where the tensor $\vec{\sigma}=\left(\sigma_{1}, \sigma_{2}, \sigma_{3}\right)$ is known as the Pauli spin vector [20]. Note that the nonlinear terms of SPM and scalar XPM are weighted by a nonlinear coefficient scaled by $8 / 9$ and $4 / 3$, respectively. The vectorial XPM effects are taken into account by the traceless Hermitian matrices $\vec{p} \cdot \vec{\sigma}=\sum_{i=1}^{3} p_{i} \sigma_{i}$ and $\vec{s} \cdot \vec{\sigma}=\sum_{i=1}^{3} s_{i} \sigma_{i}$.

Neglecting the GVD terms $\left(\beta_{2 s, p}=0\right)$, the Jones- domain (2) can be translated into an equivalent equation of motion of the Stokes' vectors [21]

$$
\left\{\begin{array}{l}
\frac{\partial \vec{s}(z, t)}{\partial z}=\frac{8}{9} \gamma e^{-\alpha z}\left(\vec{p}\left(z, t-d_{s p} z\right) \times \vec{s}(z, t)\right) \\
\frac{\partial \vec{p}\left(z, t-d_{s p} z\right)}{\partial z}=\frac{8}{9} \gamma e^{-\alpha z}\left(\vec{s}(z, t) \times \vec{p}\left(z, t-d_{s p} z\right)\right)
\end{array}\right.
$$

where the symbol $\times$ stands for vector cross-product. Equation (3) describes the motion of the pump/probe Stokes' vectors as a local rotation around a time- and $z$-varying axis along the fiber, where the rotation speed depends on the power of the modulated channels and on the amount of walk-off among them. In the general case of OOK-modulated signals, the previously shown equation of motion does not have a closed-form solution.

As a special case, it is known [1], [12] that, when both probe and pump are CW, the Stokes' vectors $\vec{s}(z)$ and $\vec{p}(z)$ in (3) perform a rotation around a time- and $z$-independent pivot $\vec{m}=$ $\vec{s}(z)+\vec{p}(z)$. The proof is simply obtained by adding the two time-independent (3). At coordinate $z$, the rotation angle is

$$
\Psi(z)=\frac{8}{9} \gamma P_{m} \int_{0}^{z} e^{-\alpha z^{\prime}} d z^{\prime}=\frac{8}{9} \gamma P_{m} L_{\mathrm{eff}}(z)
$$

where the magnitude of the pivot $P_{m}=$ $\sqrt{P_{s}^{2}+P_{p}^{2}+2 P_{s} P_{p} \cos \theta}$ depends on the (fixed) relative polarization angle $\theta$ between $\vec{s}$ and $\vec{p}$ and on the peak powers of the probe and the pump $P_{s}$ and $P_{p}$ respectively, $L_{\text {eff }}(z)$ is the effective fiber length, and the rotation speed in (4) decreases in $z$ because of the fiber attenuation $\alpha$. Note that the two signals undergo the same rotation angle around $\vec{m}$; however, if the transmitted power is much larger on one channel, such channel practically remains fixed, since its 
Stokes' vector is almost coincident with $\vec{m}$. Finally, note that the rotation angle does not depend on the wavelength distance between pump and probe: no matter how far apart, the two $\mathrm{CW}$ channels undergo the rotation in (4).

According to such CW model, the WDM channels clearly do not depolarize. However, the CW assumption for WDM channels is reasonable only for those systems in which chromatic dispersion or channel spacing are so large that each channel is only affected by the average power of the remaining channels. Hence, the CW model fails to describe the depolarization due to channel modulation and finite walk-off.

In order to tackle such a more general case, we adopt the following carousel model as an approximate solution of (3), valid for completely polarized input pump and probe fields, with an OOK-modulated pump and a $\mathrm{CW}$ probe.

Assume at $z=0$ that the pump is ON. As in the CW model, the "carousel" of the two Stokes' vectors starts rotating around a fixed pivot $\vec{m}=\vec{s}(0)+\vec{p}(0)=P_{m} \hat{m}$, where $\hat{m}$ is a unit vector. As soon as the pump goes OFF, the carousel stops its rotation around $\hat{m}$ and resumes it only when the pump goes ON again. At $z$, the rotation angle is [6]

$$
\Psi(z, t)=\frac{8}{9} \gamma P_{m} \int_{0}^{z} e^{-\alpha z^{\prime}} p\left(t-d_{s p} z^{\prime}\right) d z^{\prime}
$$

and depends on the section of the OOK-modulated pump bits that have walked past the probe from the input to coordinate $z$, as expressed by the normalized pump power $p\left(t-d_{s p} z^{\prime}\right)$ in the above integral. It is such A time-varying rotation angle around an average angle $\langle\Psi(z, t)\rangle=(1 / 2)(8 / 9) \gamma P_{m} L_{\text {eff }}(z)$ (due to the $\mathrm{CW}$ component of the pump and predictable by the $\mathrm{CW}$ model) that causes the depolarization of the probe signal, whose DOP is therefore decreased, as we will shortly quantify. It can be easily verified from the first equation in (3) that the solution shown previously is exact in the limiting case $P_{p} \gg P_{s}$ and nonreturn-to-zero (NRZ) pump bits.

We note that with the change of variable $\tau=d_{s p} z^{\prime}$, the integral in (5), can be written as a convolution $(\otimes)$ operation

$$
\Psi(z, t)=\frac{8}{9} \gamma P_{m}[p(t) \otimes h(t)]
$$

where the walk-off filter inpulse response [22] is

$$
h(t)=\frac{1}{d_{s p}} e^{-\frac{\alpha t}{d_{s p}}} \prod\left(\frac{t-\frac{d_{s p} z}{2}}{d_{s p} z}\right)
$$

where $\Pi(x)$ is a gating function taking value 1 for $-0.5<x<$ 0.5 , and zero else. Note that, for simplicity, we assumed $d_{s p}>$ 0 , although the case $d_{s p}<0$ is similar [22]. The frequency response of the walk-off filter has zeros at multiples of $f_{0}=$ $1 / d_{s p} z$ due to the gating function [22], [23]. Hence, a periodic 1010 pump sequence, of a period twice the bit time $T$, should produce on the probe only an average rotation $\langle\Psi(z, t)\rangle$ and no depolarization at $z$ if $1 / 2 T$ is a multiple of $f_{0}$, i.e., if the fiber length $z$ is a multiple of $\left(2 T / d_{s p}\right)=2 L_{w}$, with $L_{w}=T / d_{s p}$

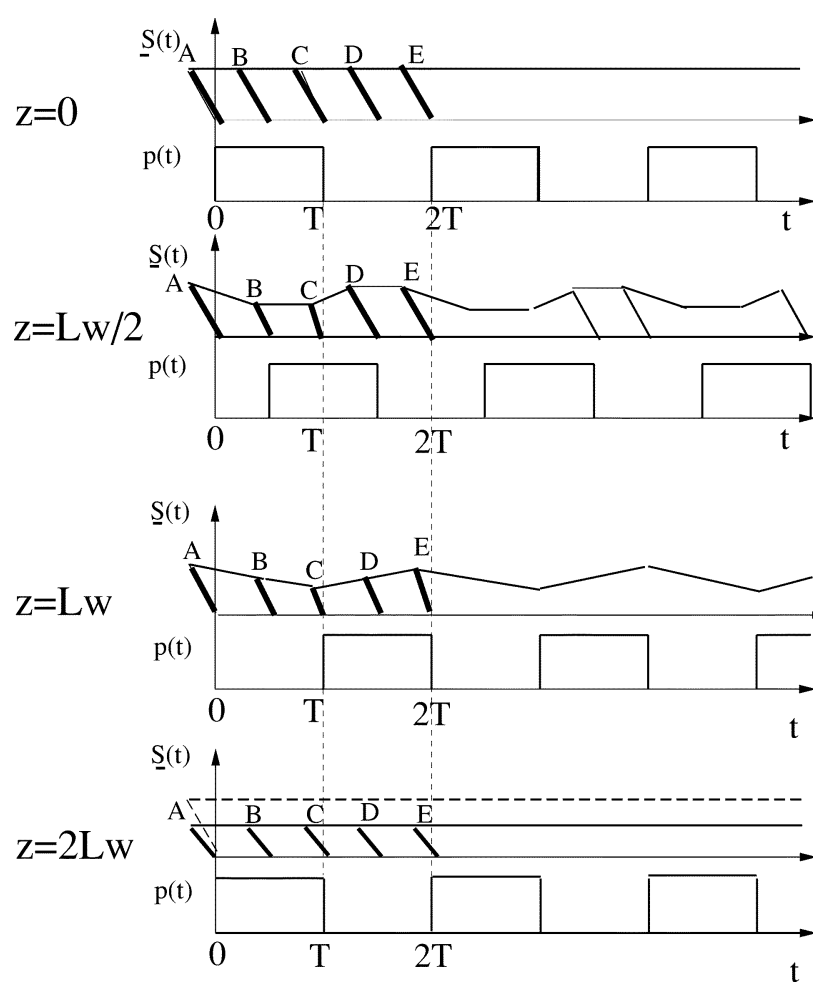

(a)

(b)

Fig. 1. Sketch of the interaction of an initially $\mathrm{CW}$ probe and a periodic square waveform pump predicted by the carousel model, at different propagation lengths. See text for a description.

being the walk-off length, i.e., the length over which the pump walks past the probe by one bit.

We visualize such a case in Fig. 1, where we represent the interaction between a linearly polarized $\mathrm{CW}$ probe and a circularly polarized pump modulated by the periodic alternation of mark and space bits, in the case $P_{p} \gg P_{s}$. We also neglect the attenuation. The (real) envelope of the probe $\underline{S}(z, t)$ and the normalized power $p(z, t)$ of the two propagating fields are portrayed in the time domain at multiples $z$ of half the walk-off length. Focus on the interaction between the first mark bit of the pump and the time "sections" A, B, C, D, E of the probe at times $t=0, \mathrm{~T} / 2$, $\mathrm{T}, 3 \mathrm{~T} / 2,2 \mathrm{~T}$ respectively. At $z=0$ the probe is completely polarized [Fig. 1(a)]. At $z=\left(L_{w} / 2\right), \mathrm{A}, \mathrm{D}$ and $\mathrm{E}$ are unaltered, as they have not interacted with the pump, while $\mathrm{B}$ and $\mathrm{C}$ are equally rotated by the pump [Fig. 1(b)]. At $z=L_{w}$, sections A and $\mathrm{E}$ are still unaltered, section $\mathrm{B}$ has the same rotation as at $z=\left(L_{w} / 2\right)$ since it stopped sensing the pump bit, while section $\mathrm{C}$ is the most rotated, and section $\mathrm{D}$ has the same rotation as section B since it sensed the pump bit for the same amount of time [Fig. 1(c)]: this is the $z$ coordinate at which the depolarization induced by XPM is the largest. Moving at $z=\left(3 L_{w} / 2\right)$, sections $\mathrm{A}$ and $\mathrm{E}$ start rotating while the most advanced section $\mathrm{C}$ stops sensing the pump. Finally at $z=2 L_{w}$, all probe sections have rotated by the same amount as they sensed the pump bit by the same amount of time, so that the probe is again completely polarized, as predicted by (6). If the pump period increases to $2 k T$, the same situation of total repolarization of the probe can be found at $z=2 k L_{w}$, and the maximum polarization rotation at section $\mathrm{C}$ at $z=k L_{w}$ is increased by $k$ times. Such argument can convince us that long sequences of many consecutive marks 


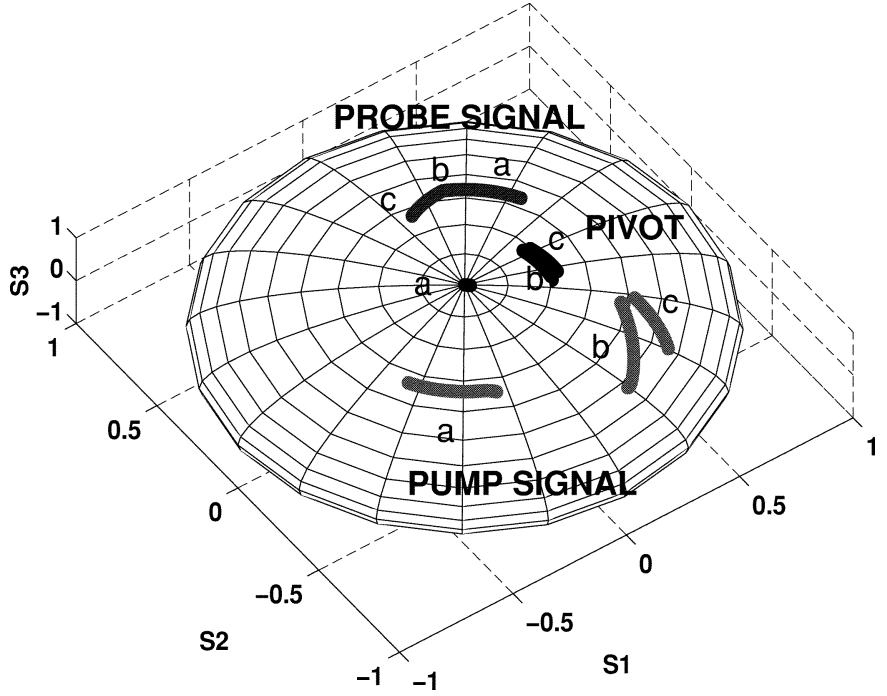

Fig. 2. SOP evolution on the Poincare sphere of an initially $\mathrm{CW}$ probe, of a square-waveform pump, and of their rotation axis in a 100-km link with $D_{c}=$ $10 \mathrm{ps} / \mathrm{nm} / \mathrm{km}, \Delta \lambda=0.4 \mathrm{~nm}$, and $L d_{s p}=4 T$. BPM solution of Manakov equation.

followed by many spaces on the pump yield the worst depolarizing case. Such point will be further explored in Section III on DOP.

We now move to the limits of our model.

We already verified that the carousel model gives the exact solution of (3) when the pump largely dominates the signal and thus remains completely polarized. However, at equal signal and pump powers, we show in Fig. 2, by direct BPM solution of the Manakov (2), the SOP evolution along $z$ (for fixed time $t$ ) in a situation of large walk-off, where the SOPs of an initially CW probe $\vec{s}(z, t)$ (dark grey points), of a pump $\vec{p}(z, t)$ modulated at $10 \mathrm{~Gb} / \mathrm{s}$ by "1010..." NRZ bit pattern (grey points) and the SOP of their "rotation axis" $\vec{s}(z, t)+\vec{p}(z, t)$ (black points) are represented on the Poincaré sphere. The two channels propagate in a $100-\mathrm{km}$ polarization maintaining fiber with parameters $D_{c}=10 \mathrm{ps} / \mathrm{nm} / \mathrm{km}, \alpha=0.2 \mathrm{~dB} / \mathrm{km}, \gamma=1.34 \mathrm{~W} / \mathrm{km}$ with equal average power of $13 \mathrm{dBm}$, spaced by $0.4 \mathrm{~nm}$ and with a walk-off of $4 \mathrm{~b}$ over the link. Starting from $z=0$, during the first mark of the pump, the two SOPs rotate around the fixed pivot (black central point) describing the two arcs $a$. Then, when the pump is off the rotation stops, as expected from the carousel model. After that, since the incoming mark bit of the pump has already been depolarized by its previous propagation, the SOPs of the probe and of the pump move, respectively, on the dark grey and grey arcs $b$, rotating around a pivot that is not fixed anymore, but moves on the black trajectory $b$. The same explanation can be given for what happens on trajectory $c$.

Note that, if the pump is not a perfectly square NRZ waveform, even in the absence of pump depolarization, the pivot $\vec{m}$ varies with time during a mark bit.

More limits of our model are the following: i) since it stems from the Manakov equation, it does not take into account the depolarization due to SPM [10]; ii) the pulse distortion due to GVD is neglected; and iii) PMD is neglected.

The effect of PMD can be understood as follows. If one thinks of a real fiber as a concatenation of randomly oriented birefrin- gent plates, then within each plate pump and probe rotate by different angles around the local birefringence vector. Hence the net effect of PMD is to randomly vary the relative polarization angle $\theta$ and the orientation of the pivot $\vec{m}$ during propagation.

\section{DOP CALCULATION}

In a WDM system, the depolarizing effects of XPM can be evaluated by numerical integration of the Manakov (2) (extended to the case of $N$ channels [1]) by means of the BPM. However, the accuracy of the BPM is paid with a long computational time, especially in long links where large powers are launched.

We provide here a faster but accurate DOP calculation based on the carousel model, which postulates that at the fiber output $z=L$ the SOP of the probe depicts in time a circular trajectory around the pivot, with a rotation angle that swings in time around an average value $\langle\Psi(L, t)\rangle$ by an amount $\Delta \Psi(L, t)=$ $\Psi(L, t)-\langle\Psi(L, t)\rangle$, whose expression is obtained from (6). Without loss of generality, we choose a reference frame of the Stokes' space in which the pivot is aligned with the third Stokes' axis $S_{3}$, and the component of the average probe output SOP along $S_{2}$ is zero, so that we can express the time-dependent output probe SOP as

$$
\hat{s}(L, t)=\frac{\vec{s}(L, t)}{P_{s}}=\left[\begin{array}{c}
\sin \theta_{s} \cos \Delta \Psi(L, t) \\
\sin \theta_{s} \sin \Delta \Psi(L, t) \\
\cos \theta_{s}
\end{array}\right]
$$

where the zero-mean process $\Delta \Psi(L, t)$ represents the SOP's azimuth, and $\theta_{s}$ is the angle between the probe and the pivot. Such angle can be obtained from $\theta$ and the pump-probe power ratio $\mathrm{PR}=\left(P_{p} / P_{s}\right)$ through the analytical relationship $\theta_{s}=$ $\theta-\arctan ((\sin \theta) /(P R+\cos \theta))$. Using (8) in the definition of DOP yields

$$
\mathrm{DOP}=\sqrt{1-\sin ^{2} \theta_{s}\left[1-\langle\cos \Delta \Psi(t)\rangle^{2}-\langle\sin \Delta \Psi(t)\rangle^{2}\right]}
$$

where all we need to evaluate is the time averages $\langle\cos \Delta \Psi(t)\rangle$ and $\langle\sin \Delta \Psi(t)\rangle$, with

$$
\Delta \Psi(L, t)=\frac{8}{9} \gamma P_{m}[(p(t)-\langle p(t)\rangle) \otimes(h t)]
$$

filtering of the normalized OOK pump power.

We can already draw some conclusions from such equations. First, the larger the swing angle $\Delta \Psi$, the smaller the DOP. Therefore, since the walk-off filter is a low-pass filter, the lower the pump modulation frequency, the lower the DOP, thus confirming the same observation already made about Fig. 1. Next, if pump and probe Stokes' vectors are initially aligned or counteraligned, then $\sin \theta_{s}=0$, so that the DOP is unity. Unfortunately, the presence of PMD prevents such initial alignment to be kept during propagation, so that depolarization occurs. Finally note that, by invoking the ergodic theorem [24], one can compute the time averages in (9) by using the characteristic function (CF) of the random variable (RV) $\Delta \Psi$

$$
\begin{aligned}
E\left[e^{j \Delta \Psi}\right] & =E[\cos \Delta \Psi]+j E[\sin \Delta \Psi] \\
& =\langle\cos \Delta \Psi(t)\rangle+j\langle\sin \Delta \Psi(t)\rangle
\end{aligned}
$$

where $E[\cdot]$ denotes expectation. 
If one now wants to avoid any further analysis, one may note that (10) and (9) can be used for a fast semianalytical MonteCarlo simulation of the DOP. Although such method readily provides us with the correct DOP values, we next look for explicit exact DOP formulas that highlight the functional dependencies of the DOP.

\section{A. Periodic Pump}

We start by observing that (9) can be given a closed form if the pump is a periodic $1010 . .$. modulating signal. Consider the case of a pump $p(t)$ modulated with a periodic alternation of NRZ pulses, with $\langle p(t)\rangle=1 / 2$. A periodic and skew-symmetric signal $\tilde{p}(t)=p(t)-\langle p(t)\rangle$ results, with period $2 T$. If we assume that higher order harmonics are filtered out by the walk-off filter, only the first harmonic $\left(c_{1} / 2\right) \sin \omega_{0} t$ of its Fourier series expansion contributes to $\Delta \Psi$, with $c_{1}=(4 / \pi)$, $\omega_{0}=(\pi / T)=\pi R$ the frequency of the "1010" sequence, and $R$ the bit rate. Hence, from (10), we get

$$
\begin{aligned}
\Delta \Psi(t)(L, t) & =\frac{8}{9} \gamma P_{m}\left|H\left(\omega_{0}\right)\right| \cdot \frac{c_{1}}{2} \sin \left(\omega_{0} t+\phi_{0}\right) \\
& =\Delta \Psi_{0} \sin \left(\omega_{0} t+\phi_{0}\right)
\end{aligned}
$$

where $H(\omega)$ is the frequency response of the walk-off filter, $\phi_{0}$ the phase of $H\left(\omega_{0}\right)$, and

$$
\left|H\left(\omega_{0}\right)\right| \cong \frac{1}{\sqrt{\alpha^{2}+\left(\frac{\pi}{L_{w}}\right)^{2}}}
$$

for long fibers with $L \gg(1 / \alpha)$ [22], [23]. The maximum swing angle for the probe SOP $\Delta \Psi_{0}=(8 / 9) \gamma P_{m}\left(c_{1} / 2\right)\left|H\left(\omega_{0}\right)\right|$ decreases for increasing walk-offs $D_{c} \Delta \lambda$ and increasing bit rates, until the effect of attenuation $\alpha$ becomes dominant. In other words, a larger walk-off length $L_{w}=1 /\left(D_{c} \Delta \lambda R\right)$ increases the amount of depolarization on the probe signal, as expected according to the discussion about Fig. 1. To evaluate the time-averages in (9), we expand $\cos \Delta \Psi(t)$ and $\sin \Delta \Psi(t)$ in Fourier series as

$$
\left\{\begin{array}{l}
\cos \Delta \Psi(t)=\cos \left[\Delta \Psi_{0} \sin \left(\omega_{0} t+\phi_{0}\right)\right] \\
=J_{0}\left(\Delta \Psi_{0}\right)+2 \sum_{n=1}^{+\infty} J_{2 n}\left(\Delta \Psi_{0}\right) \cos \left[2 n\left(\omega_{0} t+\phi_{0}\right)\right] \\
\sin \Delta \Psi(t)=\sin \left[\Delta \Psi_{0} \sin \left(\omega_{0} t+\phi_{0}\right)\right] \\
=2 \sum_{n=1}^{+\infty} J_{2 n+1}\left(\Delta \Psi_{0}\right) \sin \left[(2 n+1)\left(\omega_{0} t+\phi_{0}\right)\right]
\end{array}\right.
$$

where $J_{n}(\cdot)$ is the Bessel function of the first kind of order $n$. By averaging over a time much longer than $2 T$, one gets [25]

$$
\left\{\begin{array}{c}
\langle\sin \Delta \Psi(t)\rangle=0 \\
\langle\cos \Delta \Psi(t)\rangle=J_{0}\left(\Delta \Psi_{0}\right)=J_{0}\left(\frac{\frac{4}{9} \gamma P_{m} c_{1} N}{\sqrt{\alpha^{2}+\left(\frac{\pi}{L_{w}}\right)^{2}}}\right) .
\end{array}\right.
$$

If the supporting pulses of the pump are not NRZ, (13) can be easily generalized by inserting the first Fourier coefficient $c_{1}$ of the considered pulse shape in the argument of the Bessel function $J_{0}$, although the carousel model is slightly less accurate in such case, as already noted. Propagation on $N$ perfectly compensated spans has been accounted for by multiplying $\Delta \Psi_{0}$ by $N$. Moreover, if the extinction ratio $r$ of the considered system is not zero, a corrective factor $(1-r)$ that multiplies the pivot modulus $P_{m}$ must be introduced in the argument of $J_{0}$. Now using (13) in (9) gives

$$
\mathrm{DOP}=\sqrt{1-\sin ^{2} \theta_{s}\left\{1-[\langle\cos \Delta \Psi(t)\rangle]^{2}\right\}}
$$

where the dependence of the DOP on the relative pump-probe polarization angle $\theta$ is implicit in $\theta_{s}$ and $P_{m}$. From (14) and (13) we can conclude that, if polarization control of the signals $\left(\theta=\theta_{s}=0\right.$ or $\left.\theta=\theta_{s}=\pi\right)$ cannot be achieved, e.g., due to PMD, the basic countermeasure against DOP degradation is to increase the bit walk-off by further spacing the channels or by using a more dispersive fiber, so as to reduce the argument of the Bessel function. Clearly, also increasing the bit rate $R$ implies a reduction of XPM-induced DOP degradation.

\section{B. RBS-Modulated Pump}

An empirical DOP formula for the RBS case was derived in [25], based on an extension of (13). Here instead, we provide an exact DOP formula. The appendix details a method for the evaluation of the CF of any linear filtering of a pulse-amplitudemodulated signal. In our case the output $y=\Delta \Psi$ is given in (10) and the PAM symbols are binary, with $B=(4 / 9) \gamma P_{m} N$. The convolution $g(t)=p(t) \otimes h(t)$ in (20) can be made explicit with our exponential finite memory walk-off filter $h(t)$ in (7) when the supporting pulse $p(t)$ is zero-mean NRZ. Assuming $L \gg T / d_{s p}=L_{w}$, i.e., large walk-off per span, one gets

$$
g(t) \cong \begin{cases}0 & \text { if } t<0 \\ \frac{1-e^{-\frac{\alpha t}{d_{s p}}}}{\alpha} & \text { if } 0<t<T \\ \frac{e^{-\frac{\alpha t}{d_{s p}}}}{\alpha}\left(e^{\frac{\alpha T}{d_{s p}}}-1\right) & \text { if } T<t\end{cases}
$$

so that the $\mathrm{CF}$ in (20) becomes here

$$
E\left[e^{j \Delta \Psi}\right]=\frac{1}{T} \int_{0}^{T} \cos (B g(T-\theta)) \prod_{k=2}^{\infty} \cos (B g(k T-\theta)) d \theta
$$

where all terms with $k \leq 0$ disappear in the infinite product by the causality of $g(t)$. With the change of variable $t_{0}=T-\theta$, and posing $k^{\prime}=k-2$, this simplifies to

$$
\begin{gathered}
E\left[e^{j \Delta \Psi}\right]=\frac{1}{T} \int_{0}^{T} \cos \left(\frac{4}{9} \gamma P_{m} N L_{\text {eff }}\left(\frac{T_{0}}{d_{s p}}\right)\right) \\
\cdot \prod_{k^{\prime}=2}^{\infty} \cos \left(\frac{4}{9} \gamma P_{m} N L_{\text {eff }}\left(\frac{T}{d_{s p}}\right) e^{-\frac{\alpha\left(t_{0}+k^{\prime} T\right)}{d_{s p}}}\right) d t_{0}
\end{gathered}
$$

where $L_{\text {eff }}(x)=\left(1-e^{-\alpha x}\right) / \alpha$ is the effective fiber length. The infinite product can be safely truncated to $k^{\prime}=\left\lceil L / L_{w}\right\rceil$. Since $E\left[e^{j \Delta \Psi}\right]$ in (17) is real, it coincides with $\langle\cos \Delta \Psi(t)\rangle$, and thus $\langle\sin \Delta \Psi(t)\rangle=0$. Hence again the DOP formula is given by (14). A useful and tight lower bound is found by setting $t_{0}=0$ in the integrand in (17)

$$
E\left[e^{j \Delta \Psi}\right] \leq \prod_{k^{\prime}=2}^{\infty} \cos \left(\frac{4}{9} \gamma P_{m} N L_{\mathrm{eff}}\left(L_{w}\right) e^{-\alpha k^{\prime} L_{w}}\right)
$$




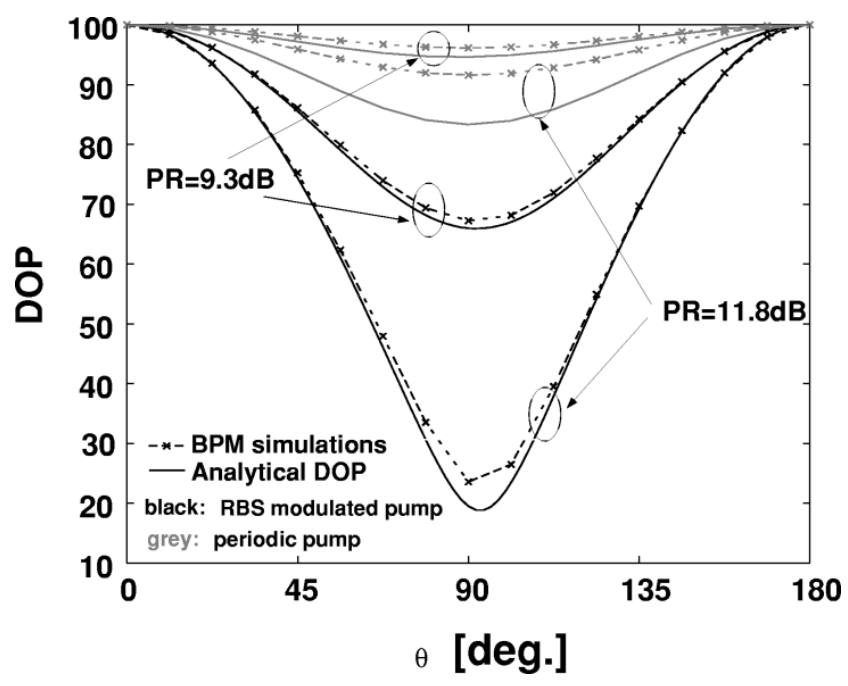

Fig. 3. DOP curves versus $\theta$ obtained with BPM numerical simulations (dashed line with crosses) and analytical DOP (14) (solid line) for two independent RBS modulated channels with NRZ pulses, propagating on a $3 \times 100-\mathrm{km}$ link of TeraLight fiber, with $\bar{P}_{s}=3 \mathrm{dBm}$, in the two cases $\mathrm{PR}=9.3 \mathrm{~dB}$ and $\mathrm{PR}=11.8 \mathrm{~dB}$. Grey lines: periodic $1010 \ldots$ pump; black lines: RBS-modulated pump.

from which it is clear that very similar considerations about the effect of system parameters as in (13) apply.

In order to validate such formulas, in Fig. 3, we compared the analytical DOP curves obtained by (14) (solid line) to those evaluated with the Manakov equation through the BPM (dashed line with crosses) when both channels are modulated with NRZ supporting pulses at $10 \mathrm{~Gb} / \mathrm{s}$, with no power on zeros $(r=0)$. The system consisted of three perfectly compensated links of TeraLight fiber $\left(\alpha=0.2 \mathrm{~dB} / \mathrm{km}, \gamma=1.68 \mathrm{~W} / \mathrm{km}\right.$, and $D_{c}=8$ $\mathrm{ps} / \mathrm{nm} / \mathrm{km}$ ) of $L=100 \mathrm{~km}$ each, with spacing $\Delta \lambda=0.8 \mathrm{~nm}$. DOP is plotted versus the angle $\theta$ between input SOPs, for an average probe power $\bar{P}_{s}=3 \mathrm{dBm}$ and two different values of power ratio $\mathrm{PR}=9.3 \mathrm{~dB}$ and $\mathrm{PR}=11.8 \mathrm{~dB}$. The grey curves refer to the periodically modulated pump, while the black curves refer to the RBS case. In the BPM simulations, the local distortion due to GVD was included $\left(\beta_{2 s, p} \neq 0\right.$ in (1)). In the RBS pump case, we observe an excellent agreement between theory and simulation. The small discrepancies around the values of minimum DOP can be attributed mostly to the assumption of a $z$-independent pivot of the carousel model (there was a walk-off of $L / L_{w}=6.4 \mathrm{~b}$ over the entire link) and in minor part to the fact that also the probe is modulated. In the periodic pump case, (14) gives a less accurate approximation of the simulated curves. We verified that this is due to the GVD distorting effect on the pump bits, which were neglected in our calculations. In the anomalous propagation in our system, the interaction of GVD and nonlinearity in fact compresses the NRZ pump pulses, so that in (13) the first Fourier harmonic of the pump should be actually given by a $c_{1}$ coefficient lower than $4 / \pi$. For this reason, the analytical results overestimate the actual probe depolarization.

In all curves, we note that depolarization is not present when the input Stokes' vectors are either aligned or counteraligned, as expected, while DOP is minimum when they are roughly orthogonal, since this implies a maximum velocity on the circular



Fig. 4. Analytical worst case DOP versus bit rate $R$ for the same system considered in Fig. 3, with RBS pump and $\mathrm{PR}=11.8 \mathrm{~dB}$.

trajectory, which is easily seen to be proportional to $P_{s} P_{p} \sin \theta$. We note a very low DOP minimum of about $20 \%$ caused by XPM at the large average pump power of $14.8 \mathrm{dBm}$ (RBS pump case), while we remark that depolarization due to PMD alone never decreases the DOP below 50\% for NRZ unchirped pulses, since the CW component cannot be depolarized by PMD [10]. Note also that (14), although valid in the pump-probe case, also applies to the worst case of an $N$-channel WDM system in which all channels have the same average power $\bar{P}_{s}$, the interfering channels are copolarized, and their vector sum has a relative polarization angle $\theta$ with respect to the probe. In such case, the number of channels $N$ depends on the power ratio $\mathrm{PR}$ as $N=10^{\mathrm{PR} / 10}+1$.

Fig. 4 shows the worst-case DOP at $\theta=\pi / 2$ versus bit rate $R$, calculated with the analytical formula (14) for an RBS modulated pump with NRZ pulses, for the previously described three-span system, both for a channel spacing $\Delta \lambda=0.8 \mathrm{~nm}$ (solid line) and for $\Delta \lambda=0.4 \mathrm{~nm}$ (dot-dashed line). In the case $\Delta \lambda=0.4$, a minimum DOP value is visible around $R \cong 13.5 \mathrm{~Gb} / \mathrm{s}$. A rough analytical approximation of such minimum-DOP value of $R$ can be obtained from (18) as $R \cong\left(\alpha /\left(D_{c} \Delta \lambda \ln 2\right)\right)$. Note that at $40 \mathrm{~Gb} / \mathrm{s}$, where the PMD distorting effects become stronger, the XPM depolarizing effects on the TeraLight produce a DOP of $70 \%$ at $\Delta \lambda=0.8 \mathrm{~nm}$, while a much lower DOP $\cong 45 \%$ at $\Delta \lambda=0.4 \mathrm{~nm}$.

To conclude this section, we show by simulation the effect on DOP of two factors that were neglected in the analysis, namely polarization-dependent loss (PDL) and PMD.

Fig. 5(a) shows the DOP for the same system used to derive Fig. 3, with a pump-probe ratio $\mathrm{PR}=11.8 \mathrm{~dB}$, but here signals had an extinction ratio of $-10 \mathrm{~dB}(r=0.1)$, and a polarizer with a PDL of either 1 or $2 \mathrm{~dB}$ and a diattenuation axis aligned either with $S_{3}$ or with $-S_{3}$ was inserted before the receiver. First focus on the solid line curve (no PDL). We note a higher value of the DOP minimum as compared with Fig. 3, due to a finite extinction ratio. Next, we note that PDL markedly breaks the symmetry of the DOP curves and shifts the minimum away from $\theta=\pi / 2$. 
(a)



(b)

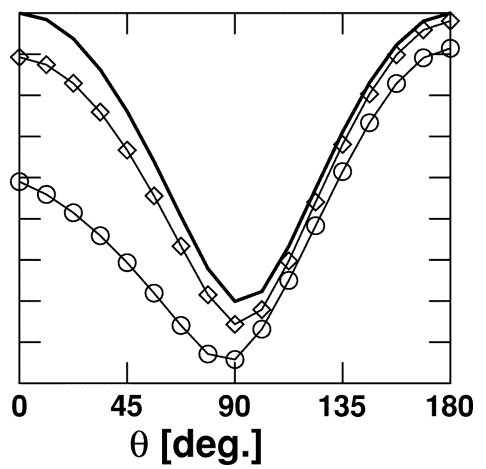

Fig. 5. DOP curves versus $\theta$ obtained by BPM simulation for the same system studied in Fig. 3 in the case of RBS-modulated channels with PR $=11.8$ dB, and with an extinction ratio $r=-10 \mathrm{~dB}$. (a) With a polarizer at the receiver and no PMD; grey dashed line: PDL $=1 \mathrm{~dB}$; black dashed line: $\mathrm{PDL}=2 \mathrm{~dB}$; solid line: without PDL. (b) Without PDL but with PMD; diamonds: DGD $=16$ ps; circles: DGD $=41$ ps; solid line: without PMD.

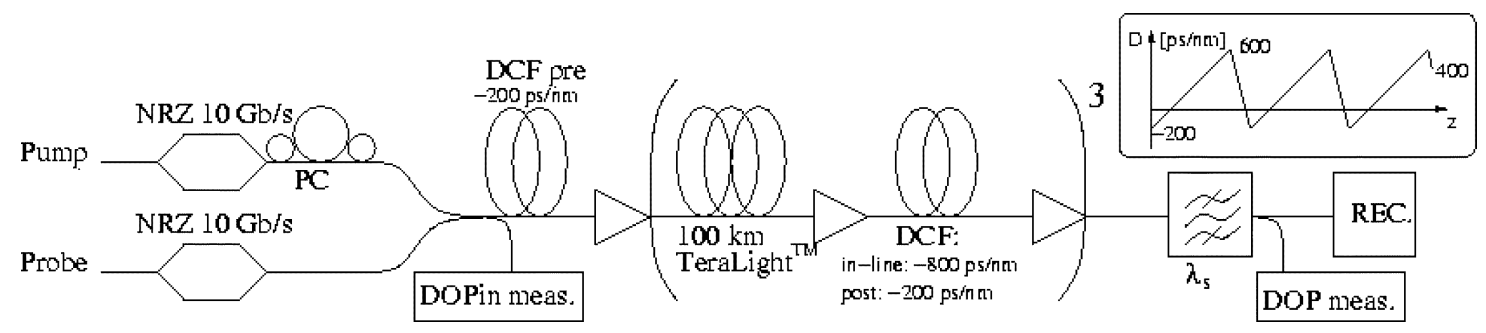

Fig. 6. Experimental setup for measurements of output probe DOP. Inset: Dispersion map.

Fig. 5(b) shows the same $3 \times 100-\mathrm{km}$ system of Fig. 5(a) without PDL and for two different fiber realizations, the first with a total differential group delay (DGD) of $16 \mathrm{ps}$ (diamonds), and the second of $41 \mathrm{ps}$ (circles). Such curves were obtained by BPM simulation of the Manakov-PMD equation [18], and took about a day of simulation time. We note that PMD further enhances the XPM-induced depolarization, the worst reduction occurring for the largest rotation speed case $\theta=0^{\circ}$, and the smallest for the lowest total DOP case $\theta=180^{\circ}$, as already noted in [11].

\section{EXPERIMENTS}

In this section, we first give some experimental results on DOP measurement that validate the new analytical formula (14). Then we show how the interaction of PMD and XPM in a twochannel system can degrade the performance of a first-order PMD compensator whose feedback signal is represented by the output probe DOP [8].

\section{A. Experimental Measurements of DOP}

We performed DOP measurements on the dispersion-managed $3 \times 100-\mathrm{km}$ link depicted in Fig. 6. The dispersion map is shown in the inset. We used TeraLight as the transmission fiber whose total measured DGD on the link is below 2 ps, so that PMD can be safely neglected. Pump and probe are spaced by $\Delta \lambda=0.8 \mathrm{~nm}$ and are NRZ modulated at $10 \mathrm{~Gb} / \mathrm{s}$ by independent $2^{15}-1$ pseudo-RBS. We performed five sets of 500 measurements of both the total input DOP and the output DOP, after filtering the probe channel, randomly changing the polarization controller (PC) each time. Fig. 7 reports the measured probe
DOP (dots) versus $\theta$ along with BPM simulation results (triangles), and the theoretical DOP curves obtained by (14) (solid line). The average probe power is fixed at $3 \mathrm{dBm}$, while the power ratio $\mathrm{PR}$ is varied for each set of measurements, being $\mathrm{PR}=6.3,9.3,11.8 \mathrm{~dB}$ in Fig. 7(a), (b), (c) respectively. The relative polarization angle $\theta$ can be easily calculated from the total measured input DOP and the power ratio PR by applying the analytical relationship DOP in $=\left(|\langle\vec{s}+\vec{p}\rangle| /\left(s_{0}+p_{0}\right)\right)=$ $\sqrt{1-(1-\cos \theta)\left((2 \mathrm{PR}) /(1+\mathrm{PR})^{2}\right)}$. The shape of the transmitted pump $p(t)$ in the experiments can be reasonably reproduced in the analysis by a raised cosine in power, with roll-off parameter $\beta=0.4 T$ and with an extinction ratio around -10 dB. In such case, the analytical DOP (14) has been calculated by using (16) with the correct pulse shape and by multiplying $P_{m}$ by a factor $(1-r)$.

We use different DOP scales to highlight the cases in which the pump power is smaller. The spread in the measurement points is mainly due to the amplifiers noise, which is the main source of depolarization when XPM is negligible. Such effect is not taken into account in theory and simulations. Some PDL, estimated in $0.5 \mathrm{~dB}$, was also present in the experiment, which justifies the slight asymmetry of the average measured DOP curve [recall Fig. 5(a)]. For increasing PR, when significant XPM effects act on the propagating signals, a reasonable match among experimental, analytical and numerical results are shown. Notice that all plots in Fig. 7(a)-(c) have the same $\mathrm{V}$-shape, and their minimum is found around $90^{\circ}$, as expected. For each considered PR value, the WDM system may be interpreted as a probe signal propagating with four, eight, or sixteen interfering channels, respectively, for PR $=6.3$, 9.3, and $11.8 \mathrm{dBm}$, where all channels have the same average power of $3 \mathrm{dBm}$ and all the interfering channels' input SOPs 


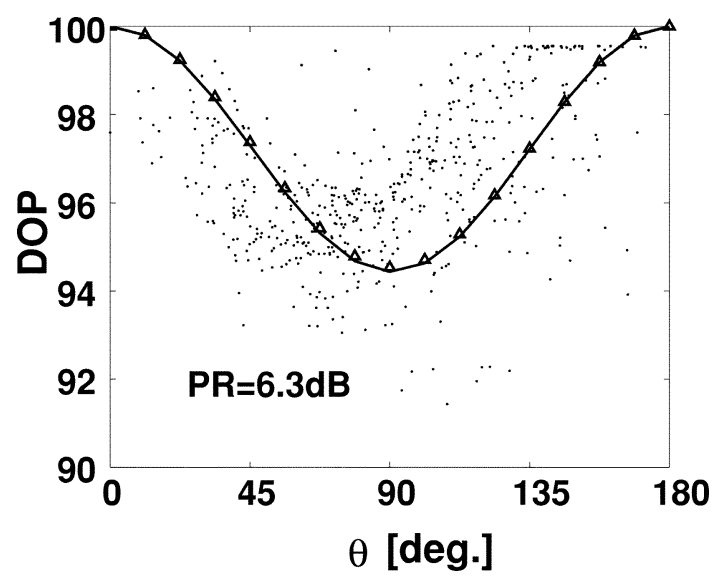

(a)

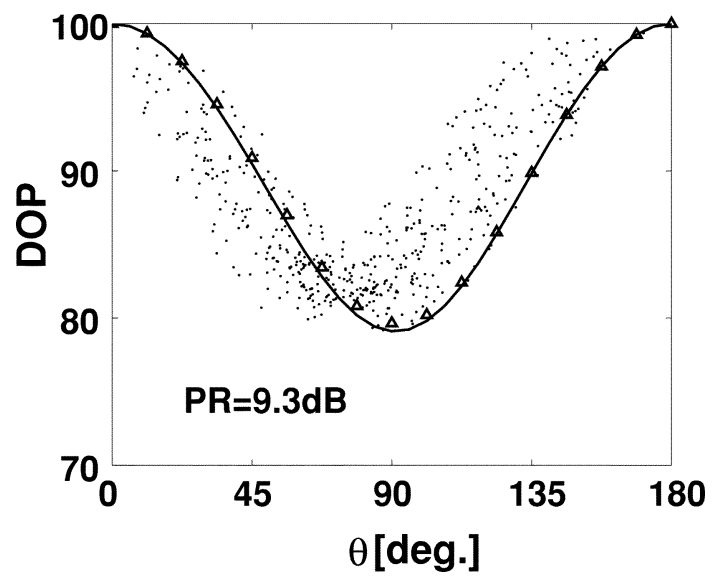

(b)

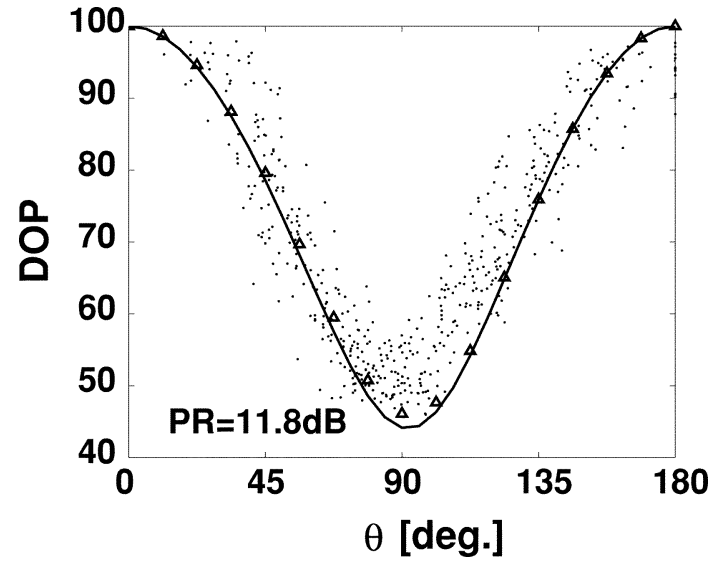

(c)

Fig. 7. Output probe DOP after three spans versus relative polarization angle $\theta$, for several pump-probe power ratios PR: measurements (dots), BPM simulations (triangles), analysis as per (14) (solid line).

are aligned as a worst case for probe DOP. Finally, in Fig. 8, BER measurements obtained for the same case of Fig. 7(c) are reported. Without giving details on the receiver, the purpose of this figure is to show that, as $\theta$ increases from $0^{\circ}$ to $180^{\circ}, \mathrm{BER}$ improves. The reason is that the dominant system impairment is here the scalar XPM-to-intensity-modulation (XPM-IM) conversion operated by GVD [14], which is maximum for aligned Stokes' vectors, and minimum for counteraligned vectors. Such two extreme cases can be regarded as scalar propagation cases of the Manakov equation, whose behavior

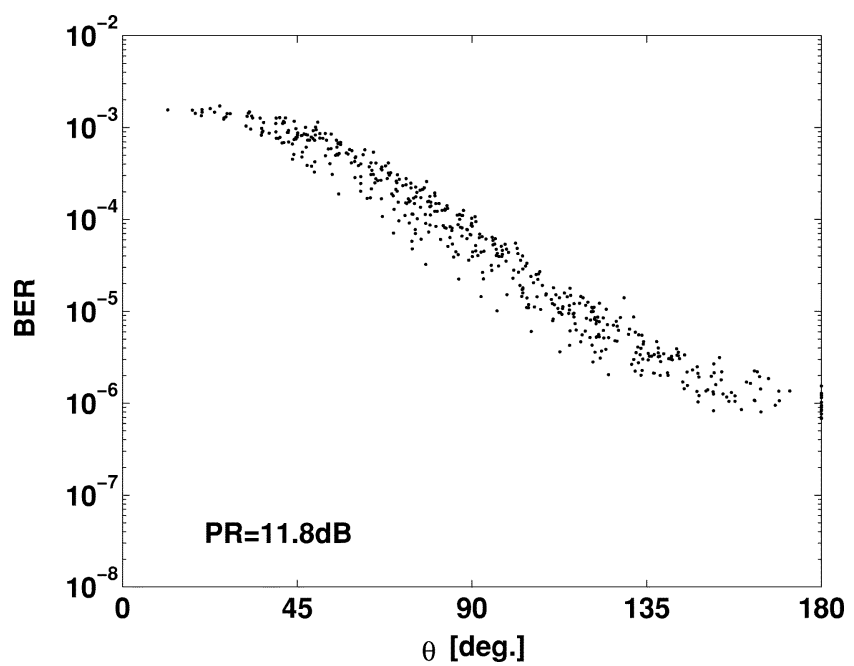

Fig. 8. Experimentally measured BER in the same case of Fig. 7(c).

can be quickly inferred from (1), where the XPM contribution in the first equation is proportional to $\left(\underline{P}^{\dagger} \underline{P}\right) \underline{S}+\left(\underline{P}^{\dagger} \underline{S}\right) \underline{P}$ : we clearly see that the second term is proportional to the first when the channels are copolarized (maximum XPM), while it disappears when they are orthogonal (i.e., $\theta=180^{\circ}$ ), giving a minimum XPM. Note that a large PDL at the receiver could change the previous conclusions, as discussed in [26].

Therefore, if input polarization control is feasible, one should always choose $\theta=180^{\circ}$ for best performance, although the presence of PMD reduces the input orthogonality and increases the XPM-IM [11].

\section{B. PMD Compensator Performance in Presence of XPM and High-Order PMD}

In this section, we quantify the performance degradation induced by XPM on a system that uses a first-order PMD compensator based on the DOP as a feedback signal [2]. Several other papers already reported the degradation in compensated systems that use different feedback signals [5], [9].

We consider the same WDM system described in the previous section, in which a high-order PMD emulator and a single-stage DOP-based PMD compensator were introduced, as shown in Fig. 9. In order to reproduce the actual interaction between highorder PMD and XPM, a distributed multisection emulator was employed, where each section consisted of a polarization controller and a PMF. One section, that introduces only first-order PMD, was inserted before the first span of TeraLight, then three sections were added at each interstage, before the chromatic dispersion compensating fiber (DCF). In this way, a condition of mixing of the PMD effects with respect to the fiber nonlinearity was achieved according to a sort of experimentally implemented BPM. In the linear regime, PMD depolarizes the output signal and its SOP changes during propagation. Consequently, the polarization angle $\theta$ between two copropagating channels is randomly perturbed by PMD during propagation. The emulator introduced an average total differential group delay of 30 ps. The PMD compensator was a single-stage compensator in which a polarization controller and a PMF were used, and a feedback loop was applied to maximize the DOP of the output 


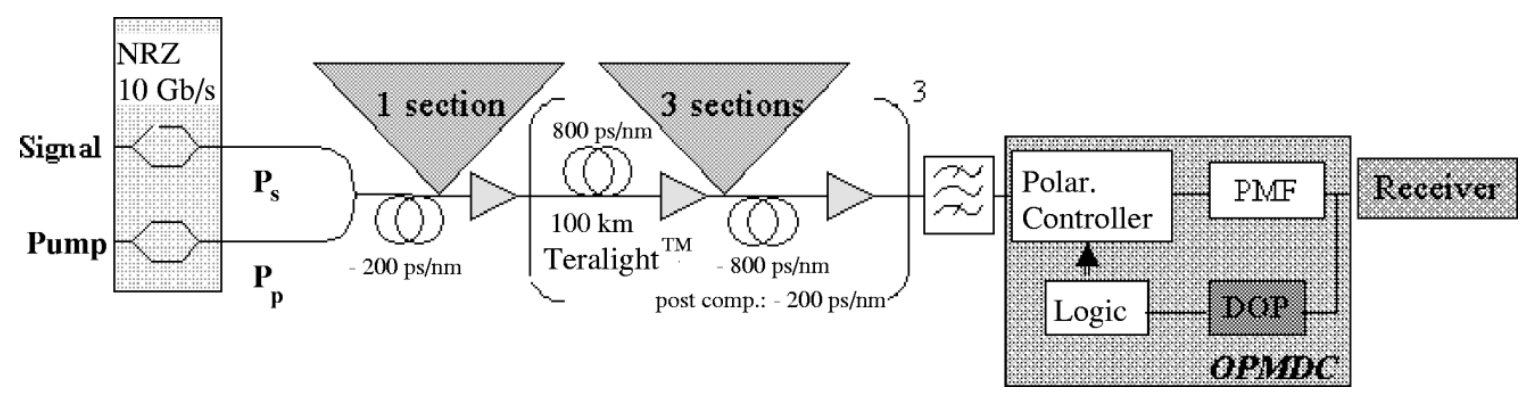

(a)



(b)

Fig. 9. Experimental setup as in Fig. 6, with insertion of a distributed PMD emulator and a first-order PMD compensator.

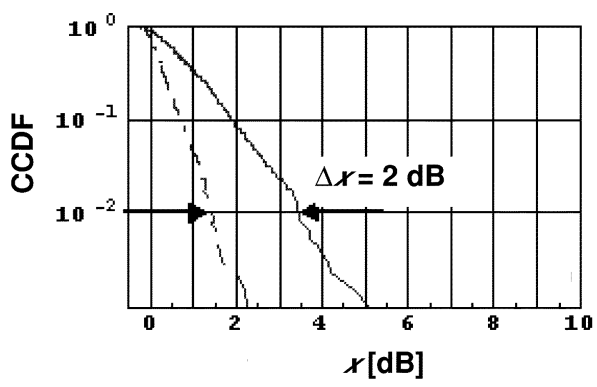

(a)

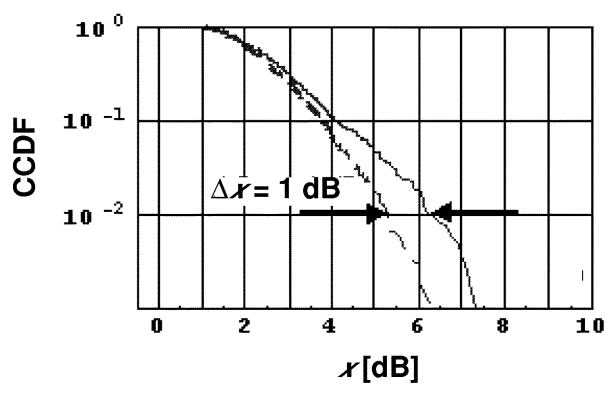

(c)



(b)

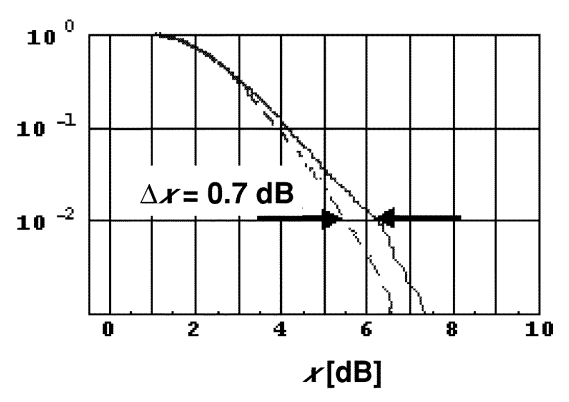

(d)

Fig. 10. CCDF of random $Q$-factor penalty $X$, with (dashed line) and without (solid line) PMD mitigation, with average DGD 30 ps, (a) in the linear regime and (b) in the nonlinear regime with $\theta=180^{\circ}$, (c) in the nonlinear regime with $\theta=0^{\circ}$, and (d) with random $\theta$.

probe [2]. The fixed DGD introduced by the compensator PMF was set at the value of $53.1 \mathrm{ps}$, which is optimum in the absence of XPM [2].

The system performance is given in Fig. 10 in terms of the complementary cumulative distribution function (CCDF) of the random $Q$-factor penalty $X$, defined as $\mathrm{CCDF}=P\{X>x[\mathrm{~dB}]\}$. The $Q$-factor penalty was measured at a constant received optical signal-to-noise ratio OSNR $=24 \mathrm{~dB}$ (over $0.1 \mathrm{~nm}$ ), with respect to the linear transmission where neither PMD nor nonlinear effects were present. The power at the preamplifier was set so as to obtain
$\mathrm{BER}=10^{-9}$ in the linear case. Fig. 10 shows the measured CCDF both in a compensated (dashed line) and in a noncompensated (solid line) system, both in the linear regime [Fig. 10(a)] and in the nonlinear regime where different values of $\theta$ have been considered, namely $\theta=180^{\circ}$ [Fig. 10(b)], $\theta=0^{\circ}$ [Fig. 10(c)], and a random $\theta$ [Fig. 10(d)]. In the latter case, the relative polarization angle was randomized over the Poincaré sphere using the PC in the probe transmitter arm. Cross- and copolarized input channels were obtained due to a polarization beam splitter and a polarization beam coupler, respectively. In each considered situation, more than 1000 
PMD conditions were drawn, so that the CCDF was accurately evaluated down to $10^{-2}$. For the random $\theta$ case, for each PMD realization a different value of $\theta$ was drawn.

In the whole experiment, the power of the probe channel, equal to $3 \mathrm{dBm}$, was kept constant so that the interplay among PMD and the nonlinear effects of SPM was negligible. The power of the pump channel was $3 \mathrm{dBm}$ in the linear regime [Fig. 10(a)], while it was switched to $12 \mathrm{dBm}$ in the nonlinear regime [Fig. 10(b)-(d)]. OSNR of the probe channel before filtering was kept constant in all cases to $24 \mathrm{~dB} / 0.1 \mathrm{~nm}$.

Note that a $Q$-factor penalty of $0.5 \mathrm{~dB}$ with only XPM was measured for the best relative polarization angle $\theta=180^{\circ}$, and of $0.8 \mathrm{~dB}$ in the worst case $\theta=0^{\circ}$, which justifies the fact that the CCDFs reach value 1 for such low penalty values. Comparing Fig. 10(b) or (c) with Fig. 10(a) in the noncompensated case, it can be observed that PMD-induced penalty, at a CCDF of $10^{-2}$ cannot be simply added to the XPM one. Moreover, a comparison among Fig. 10(b)-(d) for the noncompensated system shows that the $Q$-factor penalty is more relevant when nonlinear coupling is most efficient $\left(\theta=0^{\circ}\right)$, even if PMD mixes the relative angle $\theta$ among the propagating channels. A random choice of $\theta$ is very close to the worst case.

Now making a comparison among the performance of the noncompensated and compensated system shown in Fig. 10, a reduction of PMD compensation efficiency in presence of XPM effects is observed. In the linear case, the difference between the $Q$-factor penalty with and without PMD, respectively, is $2 \mathrm{~dB}$ for a $\mathrm{CCDF}=10^{-2}$. In the cases $\theta=0^{\circ}$ and $\theta=180^{\circ}$ such gain reduces to $1 \mathrm{~dB}$ and $1.2 \mathrm{~dB}$, respectively. When $\theta$ is randomized, the reduction of PMD compensator efficiency (down to $0.7 \mathrm{~dB}$ of gain with respect to the noncompensated system) is the most striking because a larger XPM-induced depolarization is more likely to occur, as expected.

A simplified but convincing explanation of the cooperation of PMD and XPM in the performance degradation of PMD compensated systems has been provided in [9] in terms of XPM-induced PMD-mediated intensity noise on the received probe, although we believe that a more complete system model that captures the fine details of such interaction is yet to be found.

\section{CONCLUSION}

A carousel model that allows prediction of the XPM-induced depolarization in a two-channel WDM system has been proposed. The model postulates that the Stokes' vectors of the probe and the pump perform a rotation around a fixed pivot by a bit-pattern-dependent angle that increases with the total launched power. According to the model, the worst case DOP degradation is induced by long sequences of " 1 " pump bits, while large walk-offs are found to reduce the XPM-induced depolarization. The carousel model leads to a new DOP formula based on a simple walk-off linear filtering of the pump channel, which is in good agreement with both simulations and experimental results at $10 \mathrm{~Gb} / \mathrm{s}$. Compared with the long computational time of BPM simulations, such a new formula yields a fast DOP evaluation tool, which is accurate when PMD and PDL are small. The carousel model helps identify the key physical factors that determine the XPM-induced performance degradation in OPMDC based on DOP feedback control. Finally, the performance of a first-order OPMDC in a $10-\mathrm{Gb} / \mathrm{s}$ pump-probe $3 \times 100-\mathrm{km}$ system has been experimentally evaluated, and the expected reduced efficiency in PMD compensation caused by XPM has been statistically quantified. It is verified that PMD and XPM cooperate to degrade performance. Countermeasures to reduce such degradation rely on increasing the channels' relative walk-off, as well as controlling the input SOPs so as to minimize the total DOP of the input WDM comb.

\section{APPENDIX}

In this appendix, we derive a closed-form expression of the characteristic function of any linear filtering of a pulse amplitude modulated (PAM) signal with independent symbols.

Let $x(t)=\sum_{k=-\infty}^{\infty} b_{k} p(t-k T-\theta)$ be a stationary PAM random process, with $\theta$ an RV uniform over $[0, T]$, and independent and identically distributed (IID) random symbols $b_{k}$ (independent of $\theta$ ), feeding a linear, time-invariant filter with impulse response $h(t)$. The output of such filter is $y(t)=\sum_{k=-\infty}^{\infty} b_{k} g(t-k T-\theta)$, where $g(t)=p(t) \otimes h(t)$. The CF of the RV $y(t)$ is found, for any real $s$, as [24]

$$
\Psi_{y}(s)=E\left[e^{j s y}\right]=E\left[E\left[e^{j s y} \mid \theta\right]\right]
$$

where the external expectation is performed with respect to the $\mathrm{RV} \theta$. Since the symbols are IID, then

$$
\begin{aligned}
E\left[e^{j s y} \mid \theta\right] & =\prod_{k=-\infty}^{\infty} E\left[e^{j s b_{k} g(t-k T-\theta)}\right] \\
& =\prod_{k=-\infty}^{\infty} \Psi_{b}(s g(t-k T-\theta))
\end{aligned}
$$

where $\Psi_{b}(s)$ is the common CF of the RV's $b_{k}$. Recalling that $\theta$ is uniform, we have

$$
\Psi_{y}(s)=\frac{1}{T} \int_{0}^{T} \prod_{k=-\infty}^{\infty} \Psi_{b}(s g(t-k T-\theta)) d \theta
$$

which is the sought closed-form CF. We note that since the input process is stationary and the filter time-invariant, also the output process $y(t)$ is stationary, so that any $t$ value in (19) will do, for instance $t=0$. The function $g(t)=p(t) \otimes h(t)$ can be efficiently evaluated with a fast-Fourier routine, while the external averaging can be easily evaluated by taking the average of the integrand for a finite number of $\theta$ values, uniformly sampling the interval $[0, T]$.

In the special case of binary, equally likely symbols $b_{k} \in$ $\{-B, B\}$, one gets

$$
\Psi_{b}(s)=E\left[e^{j s b}\right]=\frac{e^{j s B}+e^{-j s B}}{2}=\cos (s B)
$$

and thus

$$
\Psi_{y}(s)=\frac{1}{T} \int_{0}^{T} \prod_{k=-\infty}^{\infty} \cos (s B g(-k T-\theta)) d \theta .
$$




\section{REFERENCES}

[1] D. Wang and C. R. Menyuk, "Polarization evolution due to the Kerr nonlinearity and chromatic dispersion," J. Lightwave Technol., vol. 17, pp. 2520-2529, Dec. 1999.

[2] F. Roy, C. Francia, F. Bruyère, and D. Penninckx, "A simple dynamic polarization mode dispersion compensator," in Proc. OFC 1999, San Diego, CA, Feb. 1999, Paper TuS4, pp. 275-278.

[3] F. Heismann, D. Fishmann, and D. Wilson, "Automatic compensation of first order polarization mode dispersion compensator," in Proc. ECOC'98, Madrid, Spain, Sept. 1998, pp. 529-530.

[4] R. Noé, D. Sandel, M. Y. Dierolf, S. Honz, V. Mirvoda, A. Schöplin, C. Glingener, E. Gottwald, C. Scheerer, G. Fisher, T. Weyerauch, and W. Haase, "Polarization mode dispersion compensation at 10, 20 and 40 Gbit/s with various optical equalizers," J. Lightwave Technol., vol. 17, pp. 1602-1616, Sept. 1999.

[5] R. Khoshravani, Y. Xie, L.-S. Yan, Y. W. Song, A. E. Willner, and C. R. Menyuk, "Limitations to first order PMD mitigation compensation in WDM systems due to XPM-induced PSP changes," in Proc. OFC 2001, Anaheim, CA, 2001, Paper WAA5.

[6] Z. Pan, Q. Yu, A. E. Willner, and Y. Arieli, "Fast XPM-induced polarization-state fluctuations in WDM systems and their mitigation," in Proc. OFC 2002, Anaheim, CA, 2002, Paper ThA7, pp. 379-381.

[7] L. Möller, P. Westbrook, S. Chandrasekhar, R. Dutta, and S. Wielandy, "Setup for demonstration of cross channel induced nonlinear PMD in WDM system," Electron. Lett., vol. 37, no. 5, pp. 306-307, Mar. 2001.

[8] E. Corbel, J.-P. Thiéry, S. Lanne, S. Bigo, A. Vannucci, and A. Bononi, "Experimental statistical assessment of XPM impact on optical PMD compensator efficiency," in Proc. OFC 2003, Atlanta, GA, Feb. 2003, Paper ThJ2.

[9] J. H. Lee, K. J. Park, C. H. Kim, and Y. C. Chung, "Effects of nonlinear crosstalk in optical PMD compensation," IEEE Photon. Technol. Lett., vol. 14, pp. 1082-1084, Aug. 2002.

[10] N. Kikuchi, "Analysis of signal degree of polarization degradation used as control signal for optical polarization mode dispersion compensation," J. Lightwave Technol., vol. 19, pp. 480-486, Apr. 2001.

[11] L. Möller, S. Chandrasekhar, and L. L. Buhl, "Dependence of nonlinear depolarization on the overall polarization of PMD distorted WDM signals," in Proc. ECOC '01, Amsterdam, The Netherlands, Sept. 2001, Paper TuA3.6, pp. 214-215.

[12] B. C. Collings and L. Boivin, "Nonlinear polarization evolution induced by cross-phase modulation and its impact on transmission systems," IEEE Photon. Technol. Lett., vol. 12, pp. 1582-1584, Nov. 2000.

[13] A. Cartaxo, "Impact of modulation frequency on cross-phase modulation effect intensity modulation-direct detection WDM systems," IEEE Photon. Technol. Lett., vol. 10, pp. 1268-1270, Sept. 1998.

[14] G. Bellotti, M. Varani, C. Francia, and A. Bononi, "Intensity distortion induced by cross-phase modulation and chromatic dispersion in optical-fiber transmissions with dispersion compensation," IEEE Photon. Technol. Lett., vol. 10, pp. 1745-1747, Dec. 1998.

[15] C. R. S. Fludger, V. Handerek, and R. J. Mears, "Pump to signal RIN transfer in Raman fiber amplifiers," J. Lightwave Technol., vol. 19, pp. 1140-1148, Aug. 2001.

[16] K.-P. Ho, "Statistical properties of stimulated Raman crosstalk in WDM systems," J. Lightwave Technol., vol. 18, pp. 915-921, July 2000.

[17] G. P. Agrawal, Nonlinear Fiber Optics. New York: Academic, 1989.
[18] D. Marcuse, C. R. Menyuk, and P. K. A. Wai, "Application of the Manakov-PMD equation to studies of signal propagation in optical fibers with randomly varying birefringence," J. Lightwave Technol., vol. 15 , pp. 1735-1745, Sept. 1997.

[19] S. Huard, Polarization de la lumière. Paris, France: Masson, 1994.

[20] J. P. Gordon and H. Kogelnik, "PMD fundamentals: Polarization mode dispersion in optical fibers," Proc. Nat. Academy Sci., vol. 97, no. 9, pp. 4541-4550, Apr. 2000

[21] N. J. Frigo, "A generalized geometrical representation of coupled mode theory," IEEE J. Quantum Electron., vol. QE-22, pp. 2131-2140, Nov. 1986.

[22] A. Bononi, C. Francia, and G. Bellotti, "Impulse response of cross-phase modulation filters in multi-span transmission systems with dispersion compensation," Proc. Optical Fiber Technology 4, pp. 371-383, 1998.

[23] T.-K. Chiang, N. Kagi, M. E. Marhic, and L. G. Kazovsky, "Cross-phase modulation in fiber links with multiple optical amplifiers and dispersion compensators," J. Lightwave Technol., vol. 14, pp. 249-259, Mar. 1996.

[24] A. Papoulis, Probability, Random Variables, and Stochastic Processes, 3rd ed. New York: McGraw-Hill, 1991.

[25] A. Vannucci, A. Bononi, A. Orlandini, E. Corbel, J.-P. Thiéry, S. Lanne, and S. Bigo, "A simple formula for the degree of polarization degraded by XPM and its experimental validation," in Proc. OFC 2003, Atlanta, GA, Feb. 2003, Paper ThJ1.

[26] M. R. Philips and D. M. Ott, "Crosstalk due to optical fiber nonlinearities in WDM CATV lightwave systems," J. Lightwave Technol., vol. 17, pp. 1782-1792, Oct. 1999.

Alberto Bononi, photograph and biography not available at the time of publication.

Armando Vannucci (S'95-M'01), photograph and biography not available at the time of publication.

A. Orlandini (S'00-M'01), photograph and biography not available at the time of publication.

E. Corbel, photograph and biography not available at the time of publication.

S. Lanne, photograph and biography not available at the time of publication

S. Bigo (A'99-M'99), photograph and biography not available at the time of publication. 